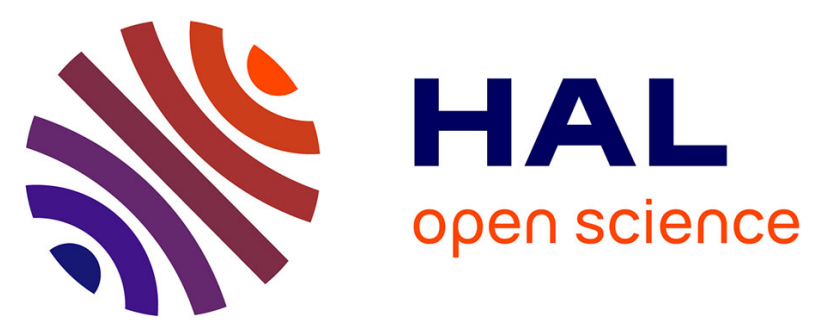

\title{
Faire preuve de sa spécificité pour se maintenir. Le travail d'entretien du territoire professionnel des rééducateurs de l'Éducation nationale (2007-2015)
}

Lorenzo Barrault-Stella, Sandrine Garcia, Anne-Élise Vélu

\section{- To cite this version:}

Lorenzo Barrault-Stella, Sandrine Garcia, Anne-Élise Vélu. Faire preuve de sa spécificité pour se maintenir. Le travail d'entretien du territoire professionnel des rééducateurs de l'Éducation nationale (2007-2015). Sociologie du Travail, 2016, 58 (3), pp.296 - 317. 10.1016/j.soctra.2016.06.003 . halshs01392747

\author{
HAL Id: halshs-01392747 \\ https://shs.hal.science/halshs-01392747
}

Submitted on 7 Jun 2021

HAL is a multi-disciplinary open access archive for the deposit and dissemination of scientific research documents, whether they are published or not. The documents may come from teaching and research institutions in France or abroad, or from public or private research centers.
L'archive ouverte pluridisciplinaire HAL, est destinée au dépôt et à la diffusion de documents scientifiques de niveau recherche, publiés ou non, émanant des établissements d'enseignement et de recherche français ou étrangers, des laboratoires publics ou privés.

\section{(ㅇ)(1) $\$$}

Distributed under a Creative Commons Attribution - NonCommercial - NoDerivatives $\mid 4.0$ 


\title{
Faire preuve de sa spécificité pour se maintenir. Le travail d'entretien du territoire professionnel des rééducateurs de l'Éducation nationale (2007-2015)
}

\author{
Demonstrating Specificity to Maintain Status. How Remedial Teachers \\ Hold Professional Territory in France's National Education System
} (2007-2015)

\author{
Lorenzo Barrault-Stella $^{\mathrm{a}, *}$, Sandrine Garcia $^{\mathrm{b}}$, Anne-Élise Vélu $^{\mathrm{c}}$ \\ a Centre de recherches sociologiques et politiques de Paris (CRESPPA), équipe Cultures et sociétés urbaines (CSU), \\ UMR 7217 CNRS, Université Paris 8 et Université Paris-Ouest Nanterre La Défense, 59-61, rue Pouchet, \\ 75849 Paris Cedex 17, France \\ ${ }^{\mathrm{b}}$ Institut de recherche sur l'éducation : sociologie et économie de l'éducation (IREDU), EA 7318 Université de \\ Bourgogne, Pôle AAFE - Esplanade Erasme, B.P. 26513, 21065 Dijon Cedex, France \\ ${ }^{\mathrm{c}}$ Institut de recherche interdisciplinaire en sciences sociales (IRISSO), UMR 7170 CNRS et Université Paris-Dauphine, \\ Paris Sciences et Lettres, Place du Maréchal de Lattre de Tassigny, 75775 Paris Cedex 16, France
}

Reçu le 30 novembre 2015 ; accepté le 30 mars 2016

Disponible sur Internet le 4 juillet 2016

\section{Résumé}

Fondé sur des archives, des entretiens et des enquêtes ethnographiques dans plusieurs écoles, cet article analyse les modalités par lesquelles un groupe professionnel peu connu, les rééducateurs de l'Éducation nationale (appelés « maîtres $\mathrm{G}$ » et chargés d'accompagner les élèves en difficulté d'apprentissage), défend une expertise spécifique et entretient son territoire dans la période contemporaine. Entre 2007 et 2012, la profession voit sa juridiction menacée politiquement par un projet visant à supprimer cette fonction, dans un contexte de concurrence exacerbée avec d'autres professionnels de la lutte contre l'échec scolaire. Les rééducateurs se défendent à travers leur organisation nationale, la Fédération nationale des associations des rééducateurs de l'Éducation nationale (FNAREN), et obtiennent une extension de leurs prérogatives à la faveur de l'alternance politique de 2012. Mais c'est surtout à travers leurs pratiques professionnelles quotidiennes qu'ils mettent en œuvre un important travail d'inférence, visant à prouver la singularité de leur

\footnotetext{
* Auteur correspondant.

Adresses e-mail : lorenzo.barrault-stella@cnrs.fr (L. Barrault-Stella), sandrine.garcia@u-bourgogne.fr (S. Garcia), anne-elise.velu@dauphine.fr (A.-É. Vélu).
} 
expertise (à mi-chemin entre celle des enseignants « ordinaires » et celle des psychologues) et l'efficacité de leurs traitements. L'observation de situations de travail, de la constitution de leurs clientèles et de leurs relations avec les autres professionnels de la lutte contre l'échec scolaire avec lesquels ils sont en concurrence potentielle, permet de souligner la manière dont ils entretiennent en acte la juridiction du groupe, son crédit et sa légitimité en mettant en avant leur spécificité professionnelle.

(C) 2016 Elsevier Masson SAS. Tous droits réservés.

Mots clés : Groupe professionnel ; Expertise ; Concurrence; Mobilisations ; Territoires ; Échec scolaire ; Psychologisation

\begin{abstract}
Drawing on archives, interviews and ethnographic surveys in several schools, this paper seeks to analyse the methods whereby a little-known professional group - remedial teachers within France's national education system (also known as 'G masters') - continues to maintain its specific expertise and professional territory. Over the period 2007-2012, the profession saw its jurisdiction and its existence come under political threat, in a context of fierce competition with other professionals dedicated to addressing educational failure. Defending their interests through their national organisation (FNAREN), remedial teachers eventually succeeded in maintaining their prerogatives as a result of the change of government in 2012 . However, it was essentially through their day-to-day practices in schools that they managed to maintain status, by seeking to demonstrate the exclusivity of their expertise (half-way between 'ordinary' teachers and psychologists) and the effectiveness of their solutions. Observing their work in situ, how they build their clientele within schools and their tangible relationships with other professionals also involved in combating educational failure, with whom they are potentially in competition, highlights the way in which they maintain the jurisdiction, credibility and legitimacy of their activity, by emphasising their own professional specificity. (C) 2016 Elsevier Masson SAS. All rights reserved.
\end{abstract}

Keywords: Professional Groups; Expertise; Competition; Mobilisation; Territories; Learning Disabilities; Psychologising

Nos diverses recherches sur les politiques éducatives en France nous ont amenés à rencontrer un groupe professionnel jusqu'alors peu connu, chargé de la rééducation des élèves en difficulté de l'enseignement maternel et primaire. Les rééducateurs de l'Éducation nationale sont des enseignants spécialisés — appelés « maîtres $G$ » — qui sont partie prenante des réseaux d'aide aux élèves en difficulté (RASED) créés en 1990. Ces réseaux d'aide complets comportent un psychologue, un « maître $\mathrm{E}$ » (chargé d'aide spécialisée à dominante pédagogique) et un « maître $\mathrm{G}$ » $^{1}$. Parfois nommés « enseignants rééducateurs », ces maîtres G sont chargés de fournir une « aide rééducative », adaptée à des difficultés dont l'origine serait davantage « affective et personnelle » que cognitive, et dans les cas où l'aide pédagogique « ordinaire » des enseignants ne fonctionnerait pas. À la différence des maîtres E, qui dispensent une aide pédagogique, ils proposent un traitement non scolaire, c'est-à-dire non intégré aux activités de classe, des difficultés scolaires des élèves, issu des théories psychanalytiques dominantes dans les années 1970. Bien que l'on ne dispose pas de données quantitatives exhaustives sur la morphologie sociale et politique de ce groupe, les rééducateurs sont pour beaucoup des femmes et sont souvent d'anciens enseignants « ordinaires » (ils appartiennent d'ailleurs toujours au corps des professeurs des écoles), usés par

\footnotetext{
${ }^{1}$ Les enseignants spécialisés sont titulaires d'un Certificat d'aptitude professionnelle pour les aides spécialisées, les enseignements adaptés et la scolarisation des élèves en situation de handicap (CAPA-SH). Ce diplôme comprend six options (de A à $\mathrm{G}$ ) qui correspondent à des catégories et à des degrés différents de difficulté ou de handicap — de l'aide pédagogique aux élèves sourds et malentendants (A) à celle apportée aux élèves présentant des troubles des fonctions cognitives (D). Les spécialisations E et G ont pour particularité d'intervenir dans le cadre de la scolarisation « ordinaire » (au sein des RASED), tandis que les autres options interviennent généralement au sein de l'enseignement spécialisé.
} 
leur métier, qui se reconvertissent dans la profession d'enseignant spécialisé $G$ par le biais d'une formation spécialisée d'une année reconnue par l'État ${ }^{2}$. Devenir maître G leur permet, outre une rémunération supplémentaire d'une cinquantaine d'euros par mois, d'améliorer leurs conditions de travail en sortant des classes, en se réappropriant leur emploi du temps et en prenant en charge de petits effectifs d'élèves ${ }^{3}$. Leur formation s'appuie principalement sur la psychologie et sur une familiarisation à des méthodes de gestion de l'échec scolaire, souvent d'inspiration ludique. Selon les textes juridiques, ces « enseignants chargés d'aides spécialisées à dominante rééducative » mobilisent principalement « la médiation du jeu à des fins d'ajustement des comportements, d'amélioration de l'autocontrôle des émotions ou des pulsions, d'instauration ou de restauration de l'estime de soi et du désir d'apprendre » des élèves en difficulté scolaire ${ }^{4}$. Concrètement, leur travail consiste à prendre en charge (généralement pour une année scolaire, parfois moins) des élèves en difficulté, individuellement ou par petits groupes, pendant les heures de cours, ce traitement constituant un substitut et non un complément aux enseignements scolaires classiques.

Ces rééducateurs constituent un groupe professionnel menacé par l'annonce ministérielle, en 2007, de la suppression des RASED dont ils sont un des principaux protagonistes. Entre 2007 et 2012, malgré diverses contestations publiques sur lesquelles nous reviendrons, de nombreux postes sont supprimés : le nombre de professionnels au sein des RASED — psychologues, maîtres E et maîtres $\mathrm{G}$ confondus - à l'échelle nationale diminue ainsi de 32,4 \% entre 2007 et 2012 (on passe de 15028 à 10125 individus). Ce sont les maîtres G qui sont les plus fortement touchés par ces suppressions, puisque cette seule profession perd près de $47 \%$ de ses effectifs en cinq ans tandis que, dans le même temps, le nombre de psychologues est maintenu ${ }^{5}$. Les rééducateurs sont au nombre de 1942 en France en 2012, selon le Ministère. Si les maîtres E sont également concernés par ces restrictions, le principe d'une aide pédagogique dans la lutte contre l'échec scolaire, concurrente donc de l'aide rééducative apportée par les maîtres $\mathrm{G}$, est réaffirmé politiquement par la mise en place gouvernementale, en 2008, du dispositif d' « aide personnalisée ». Il s'agit d'une aide assurée par les enseignants « ordinaires », au sein de la classe mais en dehors du temps scolaire classique, deux heures par semaine ${ }^{6}$. Les rééducateurs des RASED interviennent ainsi de manière à la fois complémentaire et concurrente aux actions d'autres professionnels (maîtres E, enseignants « ordinaires », psychologues, etc.) porteurs d'expertises spécifiques au sein du champ de la lutte contre l'échec scolaire (Morel, 2014).

\footnotetext{
${ }^{2}$ Sur le déclassement historique contemporain des enseignants français au principe d'une recherche, sans doute croissante, de reconversion professionnelle, voir Farges, 2011.

${ }^{3}$ Il s'agit d'une forme de mobilité « horizontale » permettant d'améliorer ses conditions de travail, comme l'a montré Howard Becker dans ses travaux sur la carrière des enseignants de l'école publique (Becker, 2006, p. 230).

${ }^{4}$ Circulaire n ${ }^{0} 2009-088$ du 17 juillet 2009 relative aux fonctions des personnels spécialisés des réseaux d'aides spécialisées aux élèves en difficulté (RASED) dans le traitement de la difficulté scolaire à l'école primaire (NOR : MENE0915410C).

${ }^{5}$ Un bilan officiel de ces restrictions de postes a été établi dans le Rapport n ${ }^{\circ} 2013-095$ de l'Inspection générale de l'Éducation nationale et de l'Inspection générale de l'administration de l'Éducation nationale et de la Recherche : Le traitement de la grande difficulté au cours de la scolarité obligatoire, novembre 2013, pp. 6670 (http://cache.media.education.gouv.fr/file/2013/27/1/2013-095_grande_difficulte_293271.pdf). Nous ne disposons pas d'informations quantitatives sur les reconversions de ces professionnels, mais une très grande majorité de ceux que nous avons rencontrés redoutaient de redevenir enseignants « ordinaires ». Les représentants de la FNAREN affirment que la plupart des maîtres G qui ont vu leurs postes supprimés ont quitté l'Éducation nationale pour se reconvertir dans le secteur social ou médical.

${ }^{6}$ Circulaire $\mathrm{n}^{0} 2008-082$ du 5 juin 2008 relative à l'organisation du temps d'enseignement scolaire et de l'aide personnalisée dans le premier degré (NOR : MENE0800496C). Ce dispositif est en concurrence avec les missions des professionnels du RASED puisqu'il suppose que la difficulté scolaire peut être prise en charge au sein de la classe par un enseignant « ordinaire ».
} 


\section{Encadré 1 : L'enquête}

Conduite de 2012 à 2015, l'enquête a été réalisée à plusieurs niveaux. D'abord, des observations ont été menées au sein de la Fédération nationale des enseignants rééducateurs (FNAREN) et d'une structure départementale de cette organisation en région parisienne, à l'occasion de réunions et de congrès militants, et des entretiens ont été réalisés avec des représentants FNAREN aux niveaux national, départemental et des établissements étudiés. Grâce aux relations de confiance nouées avec les enquêtés, la FNAREN a autorisé l'accès à une partie de ses archives contemporaines (notamment depuis 2008). Ces matériaux sont complétés par diverses sources documentaires : textes juridiques, rapports officiels, publications de la FNAREN, mémoires ou blogs, etc. Nous avons aussi opté pour deux analyses localisées (Briquet et Sawicki, 1989) - qu'on ne saurait réduire à de simples monographies tant notre démarche est caractérisée par un souci de remonter du situationnel au structurel (Schwartz, 1993) - dans deux contextes urbains de banlieue ici anonymisés. Des observations complémentaires ont également été conduites dans deux écoles (une de centre-ville relativement mixte socialement, une autre plus populaire scolarisant une importante communauté turque) d'une ville moyenne de province (un peu moins de 60000 habitants) partageant un même RASED complet.

Focalisée sur six écoles maternelles et élémentaires - dont quatre classées en Zone d'éducation prioritaire (ZEP) et deux davantage mixtes socialement - , I'enquête a débuté en 2012 , dans un contexte de relativement forte politisation des RASED. Les investigations ont été conduites dans des communes populaires de banlieue parisienne. La première correspond à deux écoles dans la partie populaire des Hauts de Seine. II y a très peu de mixité dans ces établissements, situés dans un quartier ségrégué socialement et ethniquement (immigration d'Afrique et du Maghreb, essentiellement). Les données 2010 de I'INSEE, pour les deux quartiers où sont implantées les écoles, font état d'environ $12,8 \%$ d'ouvriers, $22 \%$ d'employés, $18 \%$ d'autres professions et seulement $1,2 \%$ de cadres. La seconde zone, située en Seine-Saint-Denis, possède un RASED réparti sur quatre écoles, dont deux, relativement mixtes socialement, se trouvent dans une commune en cours de "gentrification » (I'une des plus favorisées du département). Les données 2010 de I'INSEE pour I'ensemble de la commune mentionnent 9,3\% d'ouvriers, 19,7\% d'employés et $14,7 \%$ de cadres. Dans le cadre de ces enquêtes ethnographiques, des observations ont été menées durant plusieurs années au sein de classes, des diverses réunions RASED et lors de l'accompagnement d'élèves en difficulté pour appréhender les relations de travail et la façon dont se construit le diagnostic de l'enfant en difficulté. Fiches de demande de prise en charge, entretiens approfondis, semi-directifs et comportant une dimension biographique auprès des équipes pédagogiques, des professionnels des RASED et de familles prises en charge par des maîtres $G$, constituent des matériaux privilégiés. 
Malgré ce contexte de rivalité potentielle entre professionnels et de controverses politiques autour des RASED entre 2007 et 2012, la réduction des effectifs des rééducateurs ne permet pas mécaniquement d'établir si le groupe professionnel est en déclin, car il semble paradoxalement se maintenir ${ }^{7}$. En effet, nous verrons que ces spécialistes n'ont pas disparu et que leur périmètre d'intervention semble même se renforcer dans la période récente. Comment expliquer le maintien de ce groupe professionnel au sein de l'État dans un contexte de remise en cause politique et de forte concurrence avec d'autres professionnels disposant d'expertises alternatives face au même problème de l'échec scolaire ? En croisant la sociologie des professions, les travaux relatifs à l'éducation et la sociologie de l'action publique, il s'agit d'analyser comment ce groupe professionnel entretient sa position et fait preuve de sa spécificité pour défendre son territoire à la fois politiquement et, au quotidien, dans les situations professionnelles au sein des écoles (workplace) $^{8}$. L'enjeu est alors d'étudier le travail de défense de la juridiction du groupe (au sens d'Andrew Abbott, 1988, 2003) en restituant ses relations avec les autres professionnels du secteur.

Une enquête approfondie (voir l'encadré 1) nous a permis d'étudier les manières dont les maîtres G produisent leur légitimité professionnelle et défendent leur expertise spécifique en matière de lutte contre l'échec scolaire. Les suppressions de postes au sein des RASED entre 2007 et 2012 sont analysées comme un moment particulier de remise en cause politique de la profession, où son expertise est mise en péril et où sa principale organisation — la FNAREN — négocie l'existence et l'autonomie du groupe en écartant les concurrences possibles (Chapoulie, 1973 ; Freidson, 1986). Mais les activités de résistance des rééducateurs et de préservation de leur juridiction sont surtout étudiées au prisme de leurs pratiques quotidiennes et de leurs rapports, dans les situations de travail au sein des écoles, avec les différents professionnels de la lutte contre l'échec scolaire (Chapoulie, 1984, 1987). L'enquête permet finalement d'éclairer les modalités par lesquelles un groupe professionnel menacé dans un contexte concurrentiel entretient sa juridiction, en faisant preuve de sa spécificité par la promotion d'une expertise singulière.

\section{La défense politique de la juridiction par l'organisation professionnelle}

\subsection{La construction historique d'une expertise spécifique : une prise en charge non scolaire prenant appui sur la psychanalyse}

L'histoire de la profession des maîtres $G$ s'inscrit dans un processus plus général d'élargissement des indications de la psychanalyse qui trouve son origine dans l'entre-deuxguerres, lorsque le mouvement psychanalytique cherche des territoires d'intervention qui sont la condition de son expansion (Garcia, 2011 ; Garcia et Oller, 2015). Sous l'influence d'acteurs engagés dans la cause de la psychanalyse au service de l'éducation, les Centres médico-psychopédagogiques (CMPP) voient le jour dans l'après-guerre ${ }^{9}$. Ils incarnent un projet de traitement psychanalytique des difficultés scolaires pensées comme la conséquence de troubles relationnels familiaux (Pinell et Zafiropoulos, 1978, 1983). Une telle grille de lecture est par exemple mobilisée pour analyser les échecs paradoxaux que connaissent à cette période des élèves scolarisés au lycée et socialement favorisés, le secondaire étant alors relativement fermé aux autres groupes

\footnotetext{
${ }^{7}$ Voir, par analogie, la controverse entre Eliot Freidson (1984) et Steven Brint (2006) sur le possible déclin de la profession médicale.

${ }^{8}$ Sur les usages de la sociologie du travail et des relations professionnelles dans le domaine de l'éducation, on peut lire Verdier, 2012 ; Buisson-Fenet et Verdier, 2013.

${ }^{9}$ Le premier CMPP a été créé en 1946 à Paris sous le parrainage des ministères de la Santé et de l'Éducation nationale.
} 
sociaux (Isambert-Jamati, 1985). Au début des années 1970, dans le contexte de l'unification des structures scolaires, sont créés dans les écoles des Groupes d'aide psycho-pédagogique (GAPP), qui reprennent largement le modèle des CMPP tout en l'adaptant aux contraintes du milieu scolaire ${ }^{10}$. C'est dans ces structures au sein de l'école, à mi-chemin entre traitement scolaire et prise en charge thérapeutique, qu' apparaissent les enseignants rééducateurs. Ces derniers s'organisent collectivement pour créer en 1984 une fédération, la Fédération nationale des associations des rééducateurs de l'Éducation nationale (voir l'encadré 2). Les institutions de ce type

\section{Encadré 2 : La FNAREN}

En 2009, la Fédération nationale des associations des rééducateurs de I'Éducation nationale comptait 1654 adhérents pour environ 3600 enseignants rééducateurs au total, soit presque $50 \%$ de la profession. Ce taux d'adhésion, particulièrement élevé au regard du taux moyen de syndicalisation en France (environ $8 \%$ à la même période), constitue un puissant facteur de légitimation de l'organisation dans ses rapports avec l'État. II est lié à la fois à sa situation monopolistique, aux fortes mobilisations de la profession depuis $2007^{11}$ et aux modalités concrètes d'organisation du travail des maîtres $G$ : les jeunes entrants dans la profession sont systématiquement pris en charge par un tuteur expérimenté, souvent lui-même membre de la FNAREN, type de relation interpersonnelle favorisant largement les micro-incitations à I'engagement (McAdam, 1988).

Dans son mode de fonctionnement, l'organisation professionnelle est classiquement structurée de manière à la fois souple (laissant une grande marge d'adaptation aux structures départementales) et pyramidale, avec un bureau national d'une dizaine de membres chargés de représenter l'organisation à l'échelle nationale. Majoritairement féminin, ce bureau est composé de membres qui ont moins de cinquante ans et disposent d'importantes ressources militantes (Matonti et Poupeau, 2004) acquises au cours des années 1990 lors d'expériences passées dans des organisations d'extrême gauche, antiracistes et dans des syndicats enseignants (notamment le SNUIPP, Syndicat national unitaire des instituteurs professeurs des écoles et Pegc - ces derniers étant les Professeurs d'enseignement général des collèges). Ces leaders, non rémunérés pour leurs engagements mais disposant pour mener leurs activités des importants fonds de l'organisation (plus de 160000 euros accumulés au cours du temps), représentent la profession auprès des autorités politiques. Si ces représentants du bureau national s'orientent plutôt à gauche politiquement, on ne dispose pas d'éléments sur les inclinaisons idéologiques du plus grand nombre des maîtres $G$ qui n'apparaissent pas, sur nos terrains, particulièrement politisés ou militants au-delà de la défense de leur profession.

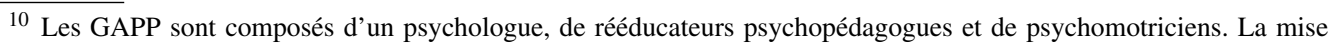
à distance du travail scolaire caractérise l'ensemble de ces acteurs et est garantie par les règles déontologiques qu'ils définissent eux-mêmes (Dorison, 2006a, p. 49).

${ }^{11}$ Le conflit peut favoriser la cohésion d'un groupe vis-à-vis de l'extérieur (Simmel, 1992).
} 
sont centrales dans la défense des groupes professionnels (Chapoulie, 1973 ; Freidson, 1986) et c'est, depuis les années 1980, la FNAREN qui fait valoir politiquement la spécificité d'une expertise des rééducateurs, distincte de celles des enseignants «ordinaires », des maîtres E et des psychologues. Si les GAPP sont remplacés en 1990 par les RASED, suite à une évaluation attestant leur manque d'efficacité sur les performances des élèves (Mingat, 1991), le groupe des rééducateurs perdure dans ce cadre jusqu'à la période récente. Ce succinct détour par l'histoire souligne la spécificité de l'expertise revendiquée de longue date par les maîtres $G$ en matière de lutte contre l'échec scolaire, fondée sur une prise en charge des difficultés scolaires non pédagogique et non scolaire (soit à l'extérieur des classes) et basée sur des formes d'appropriation de la psychanalyse.

\subsection{Résister à une remise en cause politique : faire preuve de l'expertise professionnelle du groupe et de son utilité sociale (2007-2012)}

La période 2007-2012 constitue une séquence de remise en cause politique frontale de l'existence des maîtres G. À la faveur de la victoire électorale de 2007, le gouvernement de droite annonce en effet un démantèlement des RASED et les rééducateurs sont, on l'a dit, particulièrement touchés par les suppressions de postes, avec une baisse de $47 \%$ des effectifs en cinq ans. Ces réformes sont largement liées à la recherche d'économies budgétaires dans le cadre de la Révision générale des politiques publiques (RGPP). Mais le travail d'archives révèle aussi que des doutes sur l'efficacité du traitement des rééducateurs dans la lutte contre l'échec scolaire avaient été mis en avant dans un rapport de 1997 de l'Inspection générale de l'Éducation nationale (Gossot, 1997) ${ }^{12}$. Mobilisé par le gouvernement en 2007, ce rapport blâme notamment les maîtres G pour l'adoption d'un traitement trop psychologique et individuel hors du cadre scolaire, pour des élèves pourtant signalés en difficulté d'apprentissage. Pour le dire dans les termes d'A. Abbott (1988), est reproché aux maîtres G un déficit de travail d'inférence, c'est-à-dire une insuffisante articulation du diagnostic et du traitement, qui conduit à remettre en cause la légitimité de l'expertise revendiquée par les rééducateurs.

Dans ce contexte, les maîtres G se mobilisent politiquement par le biais de la FNAREN pour consolider leur expertise dans la lutte contre l'échec scolaire et résister à leur disparition annoncée. La défense de la juridiction du groupe professionnel passe par l'usage de diverses pièces de répertoire d'action bien connues en sociologie des groupes d'intérêts (Baumgartner et al., 2009), notamment le nombre et la science (Offerlé, 1998). Dès 2007-2008, les membres du bureau national de la FNAREN tentent d'agréger des soutiens dans le secteur éducatif et, plus largement, dans l'opinion publique ${ }^{13}$. Ils sont les premiers entrepreneurs de la cause des RASED, qu'ils contribuent largement à construire, à publiciser et à politiser. Diverses publications et prises de position publiques demandent au Ministère le maintien des réseaux, et donc des effectifs des maîtres G. La mobilisation est présentée comme une défense des RASED dans leur ensemble, dans l'intérêt des enfants et non de ceux des seuls rééducateurs, évitant ainsi que l'initiative ne

\footnotetext{
12 Comme dans les cas d'autres réformes éducatives à la même période (par exemple l' « assouplissement » de la carte scolaire : Barrault-Stella, 2012), il s'agit donc de « la reprise de projets anciennement formulés et non concrétisés politiquement » (Gaïti, 1998, p. 237), désormais endossables par les acteurs politiques dominants.

13 Leurs mobilisations illustrent ainsi combien « des professionnels déploient, via leurs instances représentatives, des trésors insoupçonnés d'énergie pour tenter de modifier stratégiquement cette image auprès du public, de l'État, des autres groupes professionnels côtoyés dans le cadre quotidien de l'activité et, enfin, aux yeux de leurs propres membres » (Mathieu-Fritz et Bercot, 2008, p. 12).
} 
soit considérée comme corporatiste ou particulariste. Les représentants nationaux de la FNAREN sont à l'initiative de la création du « collectif RASED », regroupant la plupart des organisations professionnelles du secteur éducatif, dont des syndicats enseignants ${ }^{14}$, des syndicats d'autres catégories de personnels de l'institution scolaire ${ }^{15}$, divers mouvements pédagogiques ${ }^{16}$ ainsi que la principale association de parents d'élèves, la Fédération des conseils de parents d'élèves (FCPE). Sans céder à une forme d'illusion héroïque rétrospective (Dobry, 2009, pp. 45-94), si la FNAREN rallie avec succès tous ces acteurs à la cause des RASED, c'est parce qu'elle bénéficie dans le secteur éducatif de l'absence de soutien du gouvernement sur cette question. Aucune mobilisation et aucune prise de position en faveur de la réforme de démantèlement ne sont observables ${ }^{17}$. Seule la Fédération des parents d'élèves de l'enseignement public (PEEP), principale organisation de parents d'élèves orientée à droite politiquement, ne répond pas à l'appel de la FNAREN — sans pour autant s'y opposer publiquement. Les autres professionnels de la lutte contre l'échec scolaire (enseignants « ordinaires », psychologues, etc.) ne profitent pas de ce contexte politique pour se débarrasser des concurrents que constituent les rééducateurs, non seulement car ces derniers présentent leur mobilisation dans l'intérêt des enfants mais surtout, on le verra, parce qu'ils constituent souvent, dans les écoles, des soutiens importants pour leur travail. Dans le secteur éducatif à cette période, se mobiliser contre les suppressions de postes au sein des RASED devient le symbole d'une critique plus large contre la politique éducative du gouvernement impulsée par N. Sarkozy.

De 2008 à 2012, le collectif RASED impulse de nombreuses manifestations (par exemple la manifestation « 3000 bougies » devant le siège parisien du Parti socialiste, en 2010). Un « Forum des RASED » est organisé chaque année avec l'intervention de divers professionnels de l'éducation : universitaires, représentants d'associations de parents d'élèves, mouvements pédagogiques, etc. ${ }^{18}$. La FNAREN déploie un important travail de communication dans la «communauté éducative », à travers les thématiques de son congrès annuel, les publications des deux revues professionnelles de l'organisation (Envie d'école et L'Erre), ou encore en diffusant chaque année dans les écoles des plaquettes d'information sur les missions et l'utilité des rééducateurs. L'organisation réalise aussi un film documentaire très favorable à leur travail avec les élèves en difficulté, $U n$ parmi les autres, projeté en 2011 dans 490 salles à travers la France ${ }^{19}$. Lors des diffusions, des intervenants ouvrent le débat qui est animé par des rééducateurs, des universitaires ou des représentants de parents d'élèves (FCPE). Outre ce travail sur l'image publique du groupe, les membres du bureau de la FNAREN font du « lobbying » dans les milieux politiques. Entre 2008 et 2011 , ils obtiennent plusieurs audiences auprès des groupes parlementaires de gauche et du centre ainsi qu'auprès de députés et de sénateurs, emportant un ralliement très général de l'opposition du

\footnotetext{
${ }^{14}$ Comme la Confédération générale du travail Éducation (CGT-Educ'Action), le Syndicat général de l'éducation nationale (SGEN-CFDT), le Syndicat des enseignants (SE-UNSA).

${ }^{15}$ Syndicat de l'inspection de l'éducation nationale (SIEN-UNSA) ; Syndicat national des personnels de l'inspection (SNPI-FSU).

${ }^{16}$ Institut coopératif de l'École moderne - pédagogie Freinet (ICEM), Groupement français pour l'éducation nouvelle (GFEN).

17 Il n'y a pas, dans le secteur éducatif, de contre-mobilisation visant à confiner le problème à des arènes non publiques (Chabal, 2005) ou à proposer d'autres interprétations plus positives de la réforme.

18 Le thème du premier forum était, par exemple : «Faut-il supprimer les RASED ? ». Des professeurs en sciences de l'éducation, psychologie et philosophie, des maîtres formateurs ou encore le président de la FCPE y sont intervenus pour démontrer qu'un enseignant ne pouvait assumer seul la charge de la difficulté scolaire, et ainsi défendre la juridiction des maîtres G.

19 Pierre Nicola, Un parmi les autres, production FNAREN, 2011.
} 
moment à la cause des RASED à la faveur des clivages propres au champ politique. Ce faisant, la FNAREN se pose en interlocuteur incontournable pour les autorités politiques et, si l'organisation ne parvient pas à éviter de massives suppressions de postes jusqu'en 2012, cela donnera à ses représentants un certain écho ministériel après l'alternance.

Surtout, dans cette défense politique de la juridiction du groupe, la FNAREN met en œuvre un important travail d'inférence visant à prouver l'efficacité des solutions proposées par les maîtres $\mathrm{G}$ dans le traitement des élèves diagnostiqués comme en difficulté. L'enjeu est de souligner publiquement (aux parents d'élèves, dans le secteur éducatif, etc.) et aux autorités politiques que les rééducateurs disposent d'une expertise singulière, distincte de celle des autres professionnels, qui produit des effets positifs en matière de lutte contre l'échec scolaire. Pour ce faire, la FNAREN tente de légitimer l'expertise des maîtres G, et ainsi de faire preuve de leur utilité sociale, en recourant à la science. À partir de 2008, le conseil scientifique de l'organisation, composé de vingtsix psychiatres, psychologues ou psychanalystes sur quarante-quatre membres (dont quelques sociologues connus du grand public comme Alain Touraine ou Edgar Morin ${ }^{20}$ ), est régulièrement mobilisé pour intervenir publiquement. Parmi les membres de ce conseil scientifique, Jean-Jacques Guillarmé, professeur de psychopathologie à l'Université Paris Descartes, pilote en 2009 une étude réalisée à la demande du bureau national de la FNAREN (Guillarmé et Luciani, 2011), afin de prouver l'efficacité des rééducateurs, soit un procédé classique analysé par la sociologie de l'action publique (Massardier et Verdier, 2005). Produite par un membre de l'organisation publiant régulièrement dans ses revues professionnelles, cette étude scientifique montre la supériorité de la remédiation proposée par les maîtres $\mathrm{G}$ par rapport à d'autres, comme l'aide pédagogique personnalisée dispensée par les enseignants « ordinaires » hors du temps scolaire qui avait été évoquée par le Ministère pour remplacer les RASED. À partir d'entretiens avec des enseignants visant à saisir leur « ressenti », l'étude conclut que l'aide rééducative des maîtres G permet à $70 \%$ des enfants de progresser, tandis que l'aide personnalisée des enseignants hors temps scolaire ne ferait progresser que $20 \%$ des enfants (Guillarmé et Luciani, 2011, p. 15). Ces résultats attestant l'efficacité des solutions des rééducateurs contre l'échec scolaire sont ensuite publicisés par de nombreux journaux, blogs et sites spécialisés du secteur éducatif (par exemple des cafés pédagogiques). On peut en ce sens lire sur les sites de diverses organisations d'enseignants (SUD, SNUIPP, etc.) ou de parents d'élèves (FCPE) que « les RASED sont efficaces, selon une étude de Paris Descartes ». L'organisation professionnelle travaille ainsi l'image publique du groupe afin de prouver l'efficacité de son expertise professionnelle et, plus largement, son utilité sociale. Cela passe en l'espèce par la légitimation d'une expertise singulière par la science. Ces mobilisations ne suffisent toutefois pas à éviter les nombreuses suppressions de postes au sein des RASED, tant que les rapports de force politiques n'évoluent pas et malgré les contestations publiques.

\section{3. Étendre la juridiction du groupe à la faveur de l'alternance politique : la rédaction des «fiches missions »(2012-2014)}

Bien que la plupart des postes au sein des RASED aient été supprimés avant 2012, l'alternance politique ne met pas fin à la mobilisation de la FNAREN. Dès le mois de juin 2012, le collectif RASED est reçu par le nouveau ministre socialiste de l'Éducation nationale. Les socialistes avaient apporté leur soutien à la FNAREN durant la précédente mandature et le Ministère décide

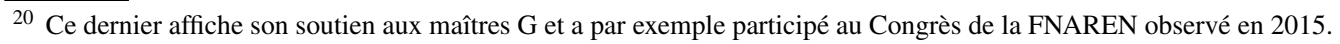


de stopper les suppressions de postes au sein des RASED, sans toutefois revenir véritablement sur celles engagées depuis $2007^{21}$. Surtout, le Ministère se lance dans une entreprise de rationalisation des périmètres d'intervention des différents professionnels de la lutte contre l'échec scolaire par l'élaboration de « fiches missions » ${ }^{22}$. Au bénéfice de sa position de leader au sein des mouvements de défense des RASED ainsi que de sa grande représentativité des rééducateurs, la FNAREN obtient d'entrée de jeu une position d'acteur central dans les négociations ministérielles sur le contenu de ces fiches.

Entre 2012 et 2014, l'élaboration des fiches missions des maîtres $\mathrm{G}$ passe par plusieurs groupes de travail au Ministère, au sein desquels les représentants de la FNAREN participent activement à la rédaction d'une nouvelle circulaire relative aux RASED. La comparaison de divers documents de travail, d'entretiens, d'articles de presse et d'archives de la FNAREN permet de restituer quelques modifications apportées au texte initial par les membres du bureau de l'organisation. Dès la première réunion en 2012, ils demandent et obtiennent par exemple la suppression du terme « scolaire » dans la phrase suivante : « [les professionnels du RASED doivent] prévenir ou/et remédier aux difficultés scolaires persistantes qui se manifestent dans l'école et qui résistent aux aides pouvant être apportées par les enseignants en classe ». Ils font également supprimer cette phrase, renvoyant à l'idée d'une intervention pédagogique : « [leur travail consiste à] repérer les difficultés de comportement des élèves et mettre en œuvre une action pédagogique pour les faire évoluer ». Leur participation à la rédaction des fiches missions leur permet de conserver un traitement non scolaire et non pédagogique des difficultés des élèves. De même, les représentants des rééducateurs insistent pour que leur rôle de «prévention » soit désormais mis en avant, alors qu'il ne l'était aucunement dans les projets ministériels initiaux, afin de pouvoir contrôler dans une certaine mesure la constitution de leur clientèle d'élèves. En effet, les entretiens biographiques menés avec les maîtres $\mathrm{G}$ montrent que ces enseignants, souvent en souffrance, espèrent une amélioration de leurs conditions de travail en devenant rééducateurs (groupe d'élèves restreint, approche émancipée des enjeux scolaires, etc.). C'est pourquoi, dans l'élaboration des fiches missions, ils tentent de peser sur la définition des types d'élèves qu'ils ont à prendre en charge, c'est-à-dire sur la définition politique de leur périmètre d'intervention. Un compte-rendu du groupe de travail de mai 2014 indique par exemple :

« Les interventions dans les situations d'urgence [c'est-à-dire a posteriori du signalement d'élèves en grande difficulté], parfois nécessaires, ne peuvent être qu' exceptionnelles. Elles ne peuvent constituer le quotidien d'aucune des trois spécialités [des RASED] et seront inefficaces si elles ne s'appuient pas sur une action durable et systémique ».

Les représentants des rééducateurs tentent en particulier d'éviter que ceux-ci soient considérés comme des «pompiers de l'urgence » - pour reprendre une expression notée lors de divers réunions et entretiens - à qui on confierait uniquement le « sale boulot », c'est-à-dire la seule prise en charge des élèves très « perturbateurs » qui « mettent le feu à l'école » (Hughes, 1962). Les rééducateurs sont soucieux de ne pas apparaître comme des « experts du comportement »au bénéfice d'une action préventive pour aider l'enfant à « retrouver le désir d'apprendre » (entretien avec la présidente de la FNAREN, juin 2014), ce qu'ils estiment ne pas pouvoir faire avec ce

\footnotetext{
${ }^{21}$ L'intention politique affichée et jusqu'alors non suivie d'effets est de « combler les suppressions de postes qui ont mis en péril les RASED ». Voir, entre autres, le rapport d'information du Sénat $n^{0} 737$ du 10 juillet 2013, p. 15.

${ }^{22}$ La loi $\mathrm{n}^{\mathrm{o}} 2013-595$ du 8 juillet 2013 d'orientation et de programmation pour la refondation de l'école de la République (NOR : MENX1241105L) prévoit que les missions de tous les personnels de l'Éducation nationale sont « modernisées » et stabilisées par écrit.
} 
type d'élèves. Ils vont donc spécifier dans les fiches missions, de manière stratégiquement non exhaustive, les «troubles » du comportement pour lesquels ils sont compétents : «immaturité, inhibition, instabilité, inappétence scolaire, difficultés de communication, mobilisation de la pensée... ». À l'occasion de la rédaction de la nouvelle circulaire relative aux RASED entre 2012 et 2014, les représentants des maîtres G parviennent donc à faire valoir une définition extensive de leurs prérogatives - non limitée à une intervention auprès des élèves les plus « perturbateurs » — et obtiennent la reconnaissance d'un périmètre d'action qui les autorise non seulement à se maintenir, mais aussi à étendre leur juridiction par rapport à celle observable avant 2007.

La satisfaction politique des demandes de groupes d'intérêt comme la FNAREN n'est possible que dès lors qu'ils formulent leurs demandes dans des formes valorisées par les agents de l'État (Courty, 1992). Ainsi, non seulement les protestations publiques ont cessé depuis l'alternance de 2012, mais surtout les leaders nationaux de la FNAREN n'évoquent que l'intérêt des élèves (et non ceux de leur groupe professionnel) dans les négociations au Ministère. En outre, pendant les deux années d'élaboration des fiches missions, les représentants des rééducateurs poursuivent le travail d'inférence. Ils multiplient les prises de position publiques rappelant l'efficacité des traitements proposés par les maîtres $\mathrm{G}$ contre l'échec scolaire, en écartant la concurrence que pourrait introduire une généralisation du dispositif de l'aide personnalisée distillée par les enseignants « ordinaires ». Par exemple, suite à la publication du Rapport ministériel pour la refondation de l'école, la FNAREN explique publiquement :

«Le dispositif d'aide personnalisée, qui a été depuis [les suppressions de postes au sein des RASED] mis en place, a clairement démontré son incapacité à améliorer significativement la situation des élèves les plus en difficulté à l'école » (FNAREN, communiqué de presse du 11 octobre 2012).

La consolidation de leur expertise en matière de lutte contre l'échec scolaire passe ainsi par la délégitimation de traitements concurrents. Toutefois, les rééducateurs ne critiquent que les remédiations alternatives proposées par le gouvernement à partir de 2007, et non les professionnels qui en ont la charge - les enseignants « ordinaires », avec lesquels ils entretiennent des relations positives depuis leur engagement convergent dans la cause des RASED ${ }^{23}$. Ils expliquent que les enseignants ne sont pas formés et ne disposent pas des moyens pour prendre en charge la grande difficulté scolaire au sein de leurs classes, ce qui leur permet de protéger leur territoire d'intervention tout en défendant les conditions de travail de ces rivaux potentiels. Enfin, dans le souci de consolider les liens avec la FCPE, les représentants des rééducateurs se font également les défenseurs de la place des parents dans la nouvelle version de la circulaire, alors que le projet initial n'y faisait pas référence.

Au bout du compte, entre 2007 et 2014, les représentants de la FNAREN sont donc parvenus à consolider la juridiction des rééducateurs, malgré un rétrécissement numérique du groupe qui n'implique pas un déclin de la profession puisque son périmètre d'intervention a été étendu à l'occasion de la confection du nouveau cadre juridique pour les RASED. Le succès relatif des maîtres G malgré une mise en péril politique du groupe est lié à l'important travail d'inférence réalisé sur la période, afin de faire preuve d'une expertise efficace et distincte de celle des autres professionnels du secteur. Mais, outre les ressources du groupe, cette réussite est dépendante

\footnotetext{
${ }^{23}$ Les syndicats enseignants ne voient d'ailleurs pas d'un bon œil que l'on ajoute à leur profession une nouvelle mission d' « aide personnalisée » à moyens constants.
} 
des opportunités, et notamment des attitudes des autres intervenants dans la lutte contre l'échec scolaire (en premier lieu les enseignants « ordinaires ») qui ne sont pas entrés en concurrence politique avec les rééducateurs et n'ont pas tenté de remettre en cause leur expertise. Il faut, pour comprendre cette absence d'opposition majeure de la part des autres spécialistes, analyser les relations entre ces différents professionnels dans les situations de travail au sein des écoles.

\section{Un travail d'inférence quotidien : la production de la reconnaissance du métier dans les écoles}

Si dans la période récente les rééducateurs se sont mobilisés politiquement à travers la FNAREN, ils s'appliquent aussi et surtout à produire la reconnaissance de leur métier à travers leurs pratiques professionnelles quotidiennes, dans leurs relations au sein des établissements avec les autres protagonistes de la lutte contre l'échec scolaire. C'est à ce niveau que leur travail d'inférence est particulièrement nécessaire à l'entretien routinier de leur crédit professionnel et à la consolidation d'un périmètre d'intervention distinct de ceux des autres professionnels (Abbott, 1988). En effet, en matière de lutte contre l'échec scolaire comme dans d'autres domaines, la différenciation des traitements possibles peut induire des conflits de compétences entre professionnels, notamment «sur les droits et les responsabilités de divers métiers et catégories de personnes à propos de la définition et de la mise en œuvre des règles de conduite en usage dans les divers aspects individuels et collectifs de la vie sociale » (Hughes, 1996, p. 100). Les enquêtes ethnographiques menées au sein de plusieurs établissements scolaires permettent de déplacer le regard vers les pratiques de travail (Arborio et al., 2008) par lesquelles les maîtres G construisent leur légitimité au quotidien et font reconnaître, par les autres professionnels comme par les familles, les spécificités de leur intervention.

Au-delà des différences potentielles selon les contextes locaux ${ }^{24}$, il existe des régularités dans les manières dont ils font valoir une expertise professionnelle spécifique. Comme au niveau politique, celle-ci repose d'une part sur une prise en charge des difficultés d'apprentissage non pédagogique (par contraste avec les maîtres E) et non scolaire (soit à l'extérieur de la classe, à la différence des enseignants « ordinaires » ou de l'aide personnalisée), et d'autre part sur un traitement ludique des difficultés psychologiques liées à l'apprentissage, ce qui les distingue des psychologues qui ne font pas de prise en charge. Les observations menées dans les écoles ont permis de mettre en évidence la façon dont les rééducateurs font reconnaître par les autres professionnels, rivaux potentiels en matière d'échec scolaire, une compétence et une expertise propre qui leur donne une relative autonomie, voire, dans certains établissements particulièrement populaires, un quasi-monopole dans la prise en charge des élèves considérés comme ayant des troubles du comportement. Leur travail d'inférence entre le diagnostic et le traitement passe avant tout par une rhétorique efficace qui rencontre l'intérêt des différents protagonistes, particulièrement dans les écoles les moins dotées. Cela leur permet le plus souvent, malgré parfois des résistances et des conflits locaux, de se (faire) constituer une clientèle au sein des établissements.

\footnotetext{
24 «L'ampleur des variations opérationnelles est prévisible » dans l'action publique (Lascoumes et Le Gales, 2007, p. 40). Par exemple, dans certains contextes ruraux, les RASED existent formellement sur le papier, mais ne comptent pas de maître G. Ils semblent plus souvent complets dans des contextes urbains concentrant une forte proportion de familles populaires.
} 


\subsection{Faire valoir une expertise spécifique pour surmonter des «difficultés à "être élève" 》}

Dans leurs relations quotidiennes au sein des établissements, les maîtres $G$ s'appliquent à montrer qu'ils disposent d'une expertise singulière permettant de traiter efficacement les élèves diagnostiqués en difficulté. Aussi, lors des observations en réunions de synthèse des RASED où l'ensemble des professionnels doit « repérer » collectivement les élèves en difficulté et décider du traitement le plus approprié en fonction du diagnostic (Chamboredon, 1971) — suivi ponctuel par le psychologue hors temps scolaire ; remédiation pédagogique par le maître $\mathrm{E}$; aide rééducative par maître $\mathrm{G}$-, les rééducateurs s'attachent presque systématiquement à prouver que les difficultés de certains élèves ne sont pas « scolaires », afin de justifier leur prise en charge non pédagogique et à l'extérieur de la classe. Ce travail consiste à démontrer que les problèmes de l'élève sont avant tout des difficultés psychologiques à « être élève », qui seraient la conséquence de diverses déviances (notamment éducatives) des familles qui, sur tous les terrains observés, appartiennent très largement aux milieux populaires. Pour ce faire, ils s'appuient généralement sur les points de vue des enseignants « ordinaires » concernant les causes familiales des difficultés scolaires. Puisque ce sont des déviances parentales qui sont présentées comme expliquant les problèmes des enfants, les remédiations proposées par les rééducateurs permettraient de combler ou d'atténuer ces supposées carences éducatives par le biais d' «activités psychologisantes » et centrées sur le jeu. Le plus souvent, lorsque le suivi par un maître $G$ est préconisé, c'est que les professionnels ont collectivement estimé que l'élève avait des difficultés à « être élève », et que son comportement (agitation ou inhibition par exemple) l'empêchait d' «entrer dans les apprentissages ».

Nos observations en réunion ainsi que les documents d'archives que nous avons consultés font ressortir de nombreux cas d'enfants soupçonnés d'avoir une «perturbation de [leur] disponibilité psychique pour les apprentissages », qui serait « liée à des difficultés affectives » dues à leur situation familiale, par exemple à un divorce, un «père alcoolique » ou une « mère absente ${ }^{25}$. Durant ces réunions, les maîtres $\mathrm{G}$ sont presque systématiquement les premiers à prendre la parole après les enseignants « ordinaires », au moment de l'élaboration du diagnostic, ce qui influe fortement sur les échanges ultérieurs. Dans les écoles observées, une fois que les enseignants ont donné des informations personnelles sur les élèves et leurs familles, les maîtres $G$ ont tendance à s'en saisir immédiatement pour faire valoir un diagnostic conforme à leur expertise et justifiant leur traitement. Par exemple, au cours d'une discussion au sujet d'une famille dite « en difficulté » en 2013, l'enseignant commence son constat en expliquant que la mère est « fragile » et qu'elle a « du mal à gérer ses émotions ». Lors du rendu des livrets, celle-ci aurait «fondu en larmes » face à l'enseignant, et l'assistante sociale ajoute que la famille est en grave difficulté financière, passant prochainement devant une commission de surendettement. La maîtresse G de l'établissement se saisit alors de ces éléments et ajoute qu'elle a croisé le père la veille dans le bistrot du quartier : «il n'avait pas l'air en bon état », indique-t-elle, sous-entendant l'existence des difficultés profondes liées à l'alcool en complément des problèmes économiques de la famille. Dès lors, l'ensemble des professionnels présents a tendance à souscrire à des interprétations psychologiques de l'échec scolaire, en considérant que ces éléments personnels ont nécessairement un impact « décisif » sur les difficultés d'apprentissage de l'enfant (Bonnery, 2008; Morel, 2012). Cela les conduit souvent à un constat d'impuissance de l'école. Par exemple, lors d'une réunion en 2013, un directeur d'école s'interroge — « avec tout ce que [tel élève ayant des difficultés familiales] vit, comment voulezvous qu' on puisse faire quelque chose pour lui ?»-, puis se déclare favorable à une prise en charge

\footnotetext{
25 Projet individuel d'aide spécialisée, document interne à un RASED (2013).
} 
par le rééducateur. De même, lors de quatre réunions de synthèse RASED où la situation scolaire de quarante élèves a été étudiée par les professionnels, la situation familiale de l'enfant et ses supposées répercussions psychologiques a été évoquée pour trente-sept des élèves ${ }^{26}$. L'ensemble des professionnels semble avoir intégré le fait que les réunions des RASED n'ont pas vocation à s'intéresser uniquement aux difficultés scolaires, mais aussi et surtout à des désordres sociaux et familiaux. Il arrive alors, comme dans les cas de dix enfants sur les quarante évoqués lors de ces quatre réunions de l'automne 2013, qu' aucune discussion ne soit engagée sur leurs apprentissages ou leurs difficultés en classe, mais que seules des informations sur leur environnement familial et leurs « dispositions psychologiques » soient abordées. Chaque professionnel ajoutant sa pierre à l'édifice, l'enjeu semble de pouvoir partager le plus d'informations, parfois intimes, sur le mode de vie de ces familles populaires en vue d'établir collégialement un diagnostic nécessitant une prise en charge hors de la classe pour remédier aux difficultés «psychologiques » et «personnelles » faisant obstacle aux apprentissages, c'est-à-dire un traitement par les maîtres $G$ à l'appui des autres professionnels.

Le travail d'inférence des rééducateurs lors de ces réunions prend appui sur l'articulation de savoirs abstraits (d'inspiration psychanalytique) et pratiques (pour les adapter aux cas particuliers des élèves à traiter). Si les psychologues sont les professionnels bénéficiant d'une forte expertise psychanalytique, ils ne prennent pas les élèves en charge et se contentent de participer à l'élaboration des diagnostics, à la différence des rééducateurs qui assurent un suivi dans le temps. Lors des réunions, les protagonistes s'accordent pour considérer l'importance des savoirs psychanalytiques dans l'identification des causes des difficultés scolaires et comportementales des élèves. Mais ils estiment aussi généralement que leur traitement nécessite une prise en charge durable durant laquelle ces savoirs abstraits seront opérationnalisés par un travail concret avec l'élève. C'est précisément cette mise en pratique casuistique des savoirs psychanalytiques à l'occasion d'échanges réguliers avec les élèves en difficulté qui fait la spécificité du traitement des maîtres G. Ces derniers mettent d'ailleurs régulièrement en avant leur expérience d'anciens enseignants, revendiquant une expertise (non pédagogique à la différence des maîtres $\mathrm{E}$ ) à mi-chemin entre celle des psychologues et celle des enseignants « ordinaires ». En ce sens, les rééducateurs font état de manières de travailler et de méthodes spécifiques, reposant sur des activités psychologisantes qui permettraient à l'élève de « retourner vers les apprentissages ». Les documents de travail des rééducateurs consultés depuis 2012 mettent par exemple en avant des objectifs comme « restaurer l'estime de soi nécessaire pour affronter les craintes face aux obstacles rencontrés dans des situations de communication et d'apprentissage », ou permettre à l'élève « d'extérioriser et ainsi de mettre à distance ses émotions [...]. Lui permettre ainsi d'investir pleinement le monde de l'école, séparé de celui de sa maison ${ }^{27}$. D'après nos observations, leurs activités concrètes avec les élèves à la place du temps scolaire sont généralement centrées sur le jeu et la « communication ». Ils mettent en place des jeux divers, des jeux de société (Bonne-Paye, Uno, Dominos, Mille-Bornes, etc.), des jeux de rôles (durant lesquels les enfants et l'adulte doivent chacun jouer un rôle ; par exemple le peintre et ses apprentis ${ }^{28}$ ), des saynètes dites «psychodramatiques » (pendant lesquelles chacun doit jouer une place « transférentiellement »; par exemple, l'adulte

\footnotetext{
${ }^{26}$ Réunions de synthèse RASED dans une circonscription populaire des Hauts de Seine à l'automne 2013.

27 Projets individuels d'aide spécialisée, document de travail d'un maître G (2013).

28 Dans le film précité, Un parmi les autres, rééducateurs et enfants jouent la relation entre le maître et l'élève avec une inversion des rôles afin de permettre à l'élève d' « exorciser » ses craintes de l'échec en se montrant « sévère » avec l'enseignant.
} 
joue le père et l'enfant la mère), ou encore des dessins dits «symboligènes ${ }^{29}$. Les rééducateurs expliquent qu'ils choisissent des jeux permettant l' « expression du vécu » et la « mise en scène des émotions ». Qu'importe le jeu choisi, les élèves pourront ainsi « jouer leurs problèmes ». Par exemple, un enfant qui ne travaille pas en classe par «peur de se tromper » pourra «jouer » et expérimenter l'échec tout en vivant une « expérience de confiance ». Une maîtresse G raconte aussi, en 2014 : «j'avais un garçon qui jouait à la poupée, il jouait son problème en fait. Il avait été abandonné par sa mère ». Outre les observations de scènes de "rééducation », ce type de pratiques se retrouve dans les «projets rééducatifs » (soit des documents de travail des RASED) consultés depuis 2012. L'expertise propre des maîtres G repose ainsi sur un subtil dosage entre un recours à des savoirs psychanalytiques appliqués et une prise en charge ludique hors de la classe qui allège dans bien des cas le travail des enseignants « ordinaires ». Mais la reconnaissance de cette spécificité au sein des établissements nécessite aussi de distinguer leur compétence de celles des autres professionnels de la lutte contre l'échec scolaire.

\subsection{Se distinguer des autres professionnels des RASED}

Pour promouvoir leur juridiction au quotidien dans les écoles, les maîtres $\mathrm{G}$ doivent montrer leur différence sous le rapport des remédiations proposées par les autres protagonistes susceptibles de traiter des élèves en difficulté. Tout d'abord, au sein des RASED, les professionnels a priori en position de force sont les psychologues scolaires, qui bénéficient de la légitimité plus générale de cette profession dans ses rapports avec l'État (Le Bianic, 2013), et qui n'ont pas été touchés par les suppressions de postes prévues entre 2007 et 2012. Dans tous les contextes observés, ils prennent en charge la coordination des RASED, organisent les réunions, et sont davantage présents au sein de chaque établissement où ils sont implantés (à la différence des rééducateurs qui circulent entre plusieurs écoles). Leur expertise est proprement psychologique. Le cœur de leur travail consiste à faire passer aux enfants en difficulté des « tests psychotechniques » pour justifier des orientations scolaires (vers des classes spécialisées) et de recevoir leurs parents, pour faire de la «guidance parentale » ou pour les inciter à accepter les orientations proposées. La plupart du temps, les psychologues considèrent les rééducateurs comme des collègues, implicitement subalternes, ayant « un travail différent » qui passe par un suivi de l'enfant, contrairement à leur intervention qui porte davantage sur le rôle des parents, sur l'orientation scolaire ou sur des situations ponctuelles. L'activité des rééducateurs leur est parfois utile puisqu'elle leur permet d'obtenir plus rapidement des informations sur les élèves et leurs comportements dans des situations non scolaires. À la différence des maîtres G, ils ne prennent pas durablement en charge les élèves, et leur remédiation est ponctuelle. Il arrive quelquefois qu'ils estiment que les rééducateurs empiètent sur leur territoire d'intervention, notamment en recevant les parents. Dans ces rares cas, ils rappellent simplement que les maîtres $\mathrm{G}$ « ne sont pas des psychologues » et critiquent leur posture psychanalytique «sans formation », remettant en cause frontalement leur expertise psychologique. Dans ces quelques occasions de tensions, les rééducateurs observés ont tendance à acquiescer, mais ils insistent dans le même temps sur les spécificités de leur traitement, non seulement leur prise en charge durable mais aussi leur expérience pédagogique antérieure. Le fait

\footnotetext{
${ }^{29}$ D'inspiration psychanalytique, la notion est dérivée du concept de « castration symboligène » développé par Françoise Dolto pour désigner une « castration » positive (s'opposant à celles qui produisent essentiellement de la frustration) en cela qu'elle permettrait à l'enfant de conforter son « surmoi ».
} 
d'être d'anciens enseignants « ordinaires » les distingue des psychologues qui, par contraste, ne revendiquent aucune compétence pédagogique ${ }^{30}$.

Les relations des rééducateurs avec les maîtres E sont plus simples, car leurs missions sont clairement distinguées depuis la création des RASED. Les maîtres E proposent des remédiations pédagogiques face à l'échec scolaire en complément des enseignants « ordinaires », sans recours à la psychanalyse ou au jeu ${ }^{31}$. Aucun conflit n'a été observé avec des rééducateurs sur nos terrains. Les maîtres E sont chargés du traitement des difficultés proprement scolaires et n'empiètent sur les territoires d'action ni des psychologues, ni des rééducateurs. Lors des réunions de synthèse, ils ont très largement tendance à rester en retrait des discussions et prennent surtout en charge des élèves pour lesquels le psychologue juge que les difficultés d'apprentissage n'ont pas d'origine psychologique. Dans certains cas toutefois, lorsqu'ils estiment s'occuper d'un nombre d'élèves trop élevé, ils peuvent en réorienter certains vers les maîtres $\mathrm{G}$, comme le montre cet exemple observé lors d'une réunion de synthèse de février 2015 où un maître E justifiait l'orientation d'un élève vers le rééducateur :

« Il n'est pas élève, il a beaucoup de mal à rentrer dans ce que l'école attend de lui, [...] ce qui pose problème c'est son attitude face à la tâche. De l'entrée dans la tâche, de mener un travail à son terme, de fournir des efforts ».

Ces cas où les maîtres E, souvent surchargés, estiment que leur intervention pédagogique ne fonctionne pas, favorisent la constitution d'une clientèle pour les rééducateurs.

\subsection{Soulager les enseignants et expliquer leurs difficultés}

Comme les maîtres $\mathrm{E}$ et plus généralement les RASED, les rééducateurs constituent très souvent une ressource pour les enseignants « ordinaires », particulièrement dans les contextes dits « difficiles » comme ceux observés, où leurs conditions de travail sont mauvaises : classes surchargées, nombreux élèves en difficulté scolaire et sociale, déviances comportementales récurrentes, etc. L'intervention des maîtres $\mathrm{G}$ contribue la plupart du temps à soulager les instituteurs et cela explique leur acceptation généralement sans difficulté au sein des établissements, comme d'ailleurs le soutien politique des organisations enseignantes à la cause des RASED. Très concrètement, la prise en charge par le maître $\mathrm{G}$, en extrayant les élèves problématiques de la classe, soulage temporairement les enseignants. Une institutrice de CE1-CE2 ${ }^{32}$ dans une commune populaire de périphérie parisienne raconte par exemple, en 2015 :

«M. venait le chercher [tous les jeudis matin]. Je n'avais pas d'échange avec elle qui pouvait m'aider [une fois l'enfant revenu dans ma classe], mais ça me soulageait, j'allais enfin pouvoir travailler avec la classe le matin ».

\footnotetext{
${ }^{30}$ Lors de la rédaction des « fiches missions » au Ministère entre 2012 et 2014, l'un des enjeux pour les représentants de la FNAREN était de différencier leur activité de celle des psychologues afin de faire valoir une expertise spécifique. Par exemple, lors du congrès de la FNAREN de juin 2014, la Présidente a expliqué, dans le cadre d'un groupe de travail sur la formation des maîtres G, combien l'expérience antérieure de l'enseignement qu'ils peuvent revendiquer, à la différence des psychologues, est fondamentale dans les discussions menées au Ministère.

31 Sur les luttes autour de la définition et des usages de la compétence pédagogique au sein des établissements scolaires, voir Chapoulie, 1979 ; Dorison, 2006b.

${ }^{32}$ Les classes nommées CE1 et CE2 désignent les cours dits élémentaires de premier et deuxième niveaux, correspondant, après le cours préparatoire $(\mathrm{CP})$, aux deuxième et troisième années d'école primaire en France.
} 
Bien que l'intervention du maître $\mathrm{G}$ ne résolve pas nécessairement les problèmes de l'élève pris en charge, les conditions de travail de l'enseignant sont améliorées pendant ce temps.

Du point de vue des spécificités de leur expertise, la distinction entretenue au quotidien par les maîtres $\mathrm{G}$ entre « troubles du comportement » et « difficultés d'apprentissage » d'ordre pédagogique permet de ne pas imputer aux enseignants la responsabilité des difficultés des enfants. Dans bien des cas, les rééducateurs expliquent par des raisons extérieures (généralement d'ordre psychanalytique et familial) les déviances des élèves et donc les difficultés potentielles des enseignants à maintenir de l'ordre en classe. Dans les relations entre professionnels au sein des écoles observées (que ce soit en réunion de synthèse ou lors d'interactions plus informelles), cette question des compétences à « tenir sa classe » est centrale, permettant par exemple de distinguer les « bons » et les « mauvais » enseignants « ordinaires ». Par exemple, lors d'une discussion informelle au sujet d'un élève diagnostiqué pour des troubles du comportement, la psychologue indiquait :

«Il est mal tombé en moyenne section de maternelle, l'instit ne savait pas tenir sa classe, c'était le chaos, ça a déclenché l'escalade » (journal de terrain, février 2015, école d'une commune populaire de la banlieue parisienne).

Pour les instituteurs, l'existence des RASED en général et de la spécialité G en particulier permet de justifier leurs difficultés à traiter les troubles comportementaux des élèves et, ainsi, de ne conserver que les aspects pédagogiques de leur intervention auprès des élèves. Ils ne sauraient être remis en cause dans leur capacité à être de bons pédagogues dès lors qu'un diagnostic psychologisant explique l'échec scolaire par des causes extra-scolaires et non pédagogiques.

En outre, les maîtres $G$ ne partagent pas avec les instituteurs toute leur expertise dans la gestion des élèves problématiques. Ils invoquent notamment le «secret » des enfants pour ne pas rendre appropriables par les enseignants « ordinaires » leurs savoirs et leurs méthodes, maintenant ainsi leur spécificité par un allongement (limité) de la chaîne d'inférence qui rend leur intervention nécessaire. En ce sens, lors des séances avec les élèves comme au cours des réunions de synthèse, les rééducateurs affirment presque systématiquement que « tout ce qui se passe dans [leur] salle est un secret». Cela conduit parfois les enseignants à leur reprocher de ne pas leur fournir de «trucs » et de solutions qui pourraient les aider dans leur gestion des classes. Mais la division du travail entre ces acteurs et le « secret » mis en avant par les rééducateurs permet à ces derniers d'entretenir leur périmètre d'intervention. Ils se protègent alors d'une potentielle «co-intervention » en classe (avec l'instituteur), et conservent leur monopole sur une prise en charge hors de la classe et non pédagogique des élèves en difficulté présentant des troubles du comportement.

\section{Les variations locales et les rares conflits dans la production d'une clientèle}

Les enquêtes ethnographiques ont jusqu'ici permis de pointer des convergences au sein de plusieurs écoles dans les moyens par lesquels les maîtres $\mathrm{G}$ défendent leur expertise et entretiennent leur légitimité. On observe cependant des variations selon les établissements et les configurations locales dans leurs relations avec les autres protagonistes de la lutte contre l'échec scolaire. En particulier, comme pour beaucoup d'autres professionnels, la clientèle des rééducateurs c'est-à-dire les élèves à prendre en charge présentant des difficultés relevant de leur périmètre d'intervention — n'est jamais assurée. Il peut ne pas exister d'élèves en difficulté, ou leurs difficultés peuvent ne pas relever des rééducateurs, au bénéfice par exemple des maîtres E chargés de l'aide pédagogique ou encore du dispositif de l' «aide personnalisée » des enseignants « ordinaires » promue par le gouvernement en 2007-2008. Les maîtres G doivent donc s'appliquer à produire 
leur clientèle au sein des établissements, ce qui, le plus souvent, ne pose guère de problème du fait de la division du travail précédemment évoquée et des spécificités de l'expertise portée par les rééducateurs par rapport aux autres spécialistes. De manière générale, le fait que les enseignants « ordinaires » acceptent qu'une prise en charge par les rééducateurs soit préconisée est conditionné à de bonnes relations internes à l'établissement, ce qui est le cas sur la plupart des terrains observés.

Toutefois, il arrive que la constitution de cette clientèle suscite des résistances. Ainsi, au sein de l'établissement le plus populaire et le plus ségrégué observé dans une ville moyenne de province ${ }^{33}$, la maîtresse $\mathrm{G}$ peine au moment de l'enquête à recruter des élèves et entretient des relations conflictuelles avec l'équipe enseignante et la direction, contrairement à ce qu'elle connaît dans l'autre école dont elle a la charge — une école de centre-ville et relativement mixte socialement. Dans l'école populaire, les enseignants préfèrent les traitements pédagogiques et semblent pour le moins sceptiques quant à l'efficacité des traitements psychanalytiques revendiqués par la rééducatrice. Ils hésitent ou refusent parfois de lui adresser leurs élèves, pour éviter que ceux-ci ne prennent «du retard sur leurs apprentissages », se justifiant par la crainte d'un déclin du niveau scolaire. Surtout, depuis 2008, l'équipe enseignante de l'école, relativement soudée et dynamique, s'est pleinement saisie du dispositif de l' « aide personnalisée » qui lui permet d'accentuer le soutien proprement scolaire aux élèves en difficulté, au détriment des autres traitements disponibles. Lorsqu'ils s'appuient sur le RASED, les enseignants orientent prioritairement les élèves en difficulté vers le maître E et non vers le maître G. Aussi, si la maîtresse $\mathrm{E}$ a vu sa clientèle réduire, la rééducatrice peine fortement à obtenir des élèves. Par exemple, durant toute l'année 2013-2014, aucun enfant de CP ne lui a été confié par les enseignants ${ }^{34}$. Dans l'incapacité de trouver une clientèle au sein de cette école, la rééducatrice s'est plainte de « difficultés à trouver sa place » auprès de l'inspectrice d'académie ${ }^{35}$, son recours hiérarchique visant à importer des ressources dans sa difficile lutte quotidienne pour trouver des élèves à traiter et donc défendre sa juridiction. L'inspectrice, nouvellement arrivée, a alors organisé une réunion (observée dans le cadre de l'enquête) avec l'ensemble des professionnels de l'établissement, durant laquelle, s'appuyant sur les consignes officielles depuis 2012 quant au maintien des RASED, elle a explicitement demandé aux enseignants de faire « des efforts » pour trouver « un public » à la rééducatrice. L'équipe enseignante a donc été enjointe par sa hiérarchie de produire une clientèle pour la maîtresse $G$ en facilitant son suivi des élèves. Il est d'ailleurs possible que la rédaction des nouvelles fiches missions achevée en 2014, où la FNAREN a obtenu nationalement une définition extensive du périmètre d'intervention des rééducateurs, facilite ce type de soutien hiérarchique à la fabrique de clients pour les maîtres $\mathrm{G}$ au sein des écoles.

D'autres modes de régulation des relations entre professionnels, moins conflictuels, ont été observés dans une école populaire de banlieue parisienne. En 2013, des enseignants ont par exemple conditionné leur accord pour déléguer au maître $\mathrm{G}$ le traitement d'une « élève mutique et inhibée » au fait qu'il prenne également en charge deux autres élèves très perturbateurs, afin de « soulager » l'enseignante de la classe. Le maître G n'a eu d'autre choix que d'accepter, malgré ses réserves : « des fois on nous prend un peu pour des pompiers de l'urgence ». La constitution

\footnotetext{
${ }^{33}$ Cette école est la plus défavorisée de la commune ; les revenus y sont les plus bas et l'évitement scolaire y est très important dès le niveau élémentaire. Beaucoup d'élèves sont issus de l'immigration (notamment turque), et ils sont parfois arrivés en France récemment.

${ }^{34} \mathrm{CP}$ : cours préparatoire, première classe d'enseignement de l'école élémentaire en France.

35 Nommée Directrice académique des services de l'Éducation nationale (DASEN) depuis 2012.
} 
de la clientèle des rééducateurs n'est ainsi jamais assurée, et ils doivent parfois négocier avec les enseignants. Ces transactions entre professionnels sont variables localement et d'une année à l'autre au sein du même établissement. Ce qui reste toutefois observable indépendamment des contextes, ce sont les très faibles marges de manœuvre dont disposent les parents dans la prise en charge de leurs enfants par le RASED. Dès lors que leur fils ou leur fille est signalé comme client potentiel du maître $G$ par les enseignants, la plupart des familles populaires, qui constituent on l'a dit la très grande majorité de leur clientèle dans les différentes localités étudiées, acceptent le traitement recommandé par les professionnels. Lorsque la «proposition » leur est faite formellement, il leur est expliqué que l'enfant sera pris en charge à l'extérieur de la classe, dans un cadre "moins scolaire ». Les entretiens menés avec les parents montrent que, s'ils se soumettent globalement aux injonctions de l'école, certains regrettent parfois que leurs enfants soient privés d'apprentissages :

«Mon fils il aime trop jouer, c'est pas étonnant, ils l'envoient tout le temps jouer avec N. [la maîtresse G], et pendant ce temps il ne travaille pas. [...] Après il rentre à la maison, il veut jouer. Il ne faut pas ça ! » (père de 57 ans, sans profession, malien, habitant une commune populaire de la banlieue parisienne).

Comme l'ont observé divers travaux portant sur des contextes urbains (Millet et Thin, 2005), péri-urbains (Van Zanten, 2001) ou ruraux (Barrault-Stella, 2014), les parents des classes populaires, sans être non investis dans l'éducation des enfants, n'ont souvent d'autre choix que de se soumettre aux règles de l'institution scolaire et aux injonctions de ses professionnels, en matière de prise en charge par le RASED comme pour le reste. Pour les rééducateurs, la capacité à produire une clientèle dépend donc principalement des échanges noués avec les autres professionnels au sein de l'établissement, et non de leurs relations avec les familles qui sont maintenues à l'écart du processus de sélection.

\section{Conclusion}

De manière générale, notre recherche souligne les modalités par lesquelles le groupe des rééducateurs de l'Éducation nationale, qui est partie prenante des RASED, défend une expertise singulière et entretient son territoire professionnel dans la période contemporaine (Abbott, 1988). L'enquête menée au niveau national souligne le travail politique de l'organisation professionnelle - la FNAREN — dans le moment spécifique de mise en péril par le ministère de leur métier (2007-2012), puis l'extension de la juridiction du groupe à l'occasion de la rédaction des fiches missions (2012-2014). Mais les investigations ethnographiques dans les écoles permettent surtout de saisir la défense du groupe, dans un contexte de concurrence potentielle avec les autres professionnels intervenant face à l'échec scolaire, au prisme des pratiques professionnelles quotidiennes. Les rééducateurs mettent en œuvre un important travail d'inférence visant à prouver au sein des établissements la singularité et l'efficacité des traitements qu'ils proposent aux élèves diagnostiqués comme en difficulté d'apprentissage. Ils s'appliquent ainsi à proposer une expertise spécifique, à mi-chemin entre celle des enseignants « ordinaires » et celle des psychologues, distincte et complémentaire de celle des autres protagonistes de la lutte contre l'échec scolaire. La consolidation de la juridiction du groupe, de son crédit, de son expertise et de sa légitimité passe par l'entretien d'une spécificité professionnelle, non seulement au niveau politique lorsque le métier est menacé, mais surtout dans les activités quotidiennes de travail. 
Au-delà de son cadre empirique, cette étude propose quelques apports plus généraux. D'abord, sur le plan des pratiques de recherche, elle illustre l'intérêt d'un rapprochement entre sociologies du travail et des professions d'une part, et sociologies politique et de l'État d'autre part, ce qui nécessite des protocoles d'enquête permettant d'appréhender à la fois les milieux politico-administratifs et les situations de travail. Ensuite, en matière de sociologie des professions, le cas des rééducateurs de l'Éducation nationale, confrontés à un recul quantitatif des postes entre 2007 et 2012, suivi d'une extension du périmètre d'intervention du groupe entre 2012 et 2014, montre qu'un déclin numérique impulsé politiquement peut s'accompagner d'une contre-mobilisation autorisant finalement un maintien de la profession et de son autonomie, voire même une extension de son territoire. Certains professionnels, et pas nécessairement parmi les plus prestigieux, font ainsi preuve, face aux changements, de capacités d'adaptation qui empêchent ou limitent leur déclin annoncé (Brint, 2006). Enfin, du point de vue de la sociologie politique, ces professionnels de la lutte contre l'échec scolaire, qui sont souvent d'anciens enseignants soucieux de ne pas le redevenir, attestent que les stratégies face au déclassement social qui semble aujourd'hui caractériser les enseignants français (Farges, 2011) passent non seulement par des reconversions individuelles vers de nouvelles professions du secteur (par exemple devenir maître G) mais aussi, par la suite, par des mobilisations collectives et politiques visant à défendre durablement cette possibilité de reconversion. Le déclin social relatif des enseignants ne conduit donc pas seulement à des formes d'individualisation professionnelle (comme les cas typiques de débandade suite à l'échec de mobilisations collectives : Bourdieu et al., 1973) et à un recul des formes classiques de mobilisation politique du groupe (Sawicki, 2015). Il peut aussi produire de la politisation et de nouvelles actions collectives après leur reconversion.

\section{Références}

Abbott, A.D., 1988. The System of Professions: An Essay on the Division of Expert Labor. University of Chicago Press, Chicago.

Abbott, A.D., 2003. Écologies liées : à propos du système de professions. In: Menger, P.-M. (Ed.), Les professions et leurs sociologies. Modèles théoriques, catégorisations, évolutions. Éditions de la Maison des Sciences de l'Homme, Paris, pp. 29-50.

Arborio, A.-M., Cohen, Y., Fournier, P., Hatzfeld, N., Lomba, C., Muller, S. (Eds.), 2008. Observer le travail. Histoire, ethnographie, approches combinées. La Découverte, Paris.

Baumgartner, F.R., Berry, J.M., Hojnacki, M., Kiamball, D.C., Leech, B.L., 2009. Lobbying and Policy Change. Who Wins, Who Loses and Why. Chicago University Press, Chicago.

Barrault-Stella, L., 2012. Les résistances de la carte scolaire. Les limites du volontarisme politique dans l'action publique. Politix 98, 109-127.

Barrault-Stella, L., 2014. Les rapports à l'institution scolaire de familles populaires dans les mondes ruraux contemporains. Agora débats/jeunesse 67, 21-36.

Becker, H., 2006. Le travail sociologique. Méthodes et substance. Academic Press Fribourg, Fribourg.

Bonnery, S., 2008. Les usages de la psychologie à l'école : quels effets sur les inégalités scolaires ? Sociologies pratiques 17, 107-120.

Bourdieu, P., Boltanski, L., de Saint Martin, M., 1973. Les stratégies de reconversion. Informations sur les sciences sociales 15 (5), 61-113.

Briquet, J.-L., Sawicki, F., 1989. L'analyse localisée du politique. Lieux de recherche ou recherche de lieux ? Politix 7/8, $6-16$.

Brint, S., 2006. Saving the "Soul of Professionalism": Freidson's Institutional Ethics and the Defense of Professional Autonomy. Knowledge, Work \& Society 4 (2), 99-129.

Buisson-Fenet, H., Verdier, E., 2013. Hiérarchie des savoirs et concurrences institutionnelles : la régulation des cartes régionales des formations professionnelles initiales. Revue française de pédagogie 182, 19-30.

Chabal, J., 2005. Le risque invisible. La non-émergence d'un problème public. Politix 70, 169-195. 
Chamboredon, J.-C., 1971. La délinquance juvénile, essai de construction d'objet. Revue française de sociologie 12 (3), 335-377.

Chapoulie, J.-M., 1973. Sur l'analyse sociologique des groupes professionnels. Revue française de sociologie 14 (1), 86-114.

Chapoulie, J.-M., 1979. La compétence pédagogique des professeurs comme enjeu de conflits. Actes de la recherche en sciences sociales 30, 65-85.

Chapoulie, J.-M., 1984. Everett C. Hughes et le développement du travail de terrain en sociologie. Revue française de sociologie 25 (4), 582-608.

Chapoulie, J.-M., 1987. Les professeurs de l'enseignement secondaire. Un métier de classe moyenne. Éditions de la Maison des Sciences de l'Homme, Paris.

Courty, G., 1992. Le ministre, les affaires et son portefeuille. Politix 17, 51-78.

Dobry, M., 2009. Sociologie des crises politiques. La dynamique des mobilisations multisectorielles. Les Presses de Sciences Po, Paris [1986].

Dorison, C., 2006a. Éthique et identité collective d'un groupe professionnel. Les rééducateurs de l'Éducation nationale (1960-1990). Recherche et formation 52, 43-54.

Dorison, C., 2006b. La place de la pédagogie dans les relations entre enseignants spécialisés et enseignants des classes ordinaires : du tabou à la collaboration. Les sciences de l'éducation pour l'Ère nouvelle 38, 63-78.

Farges, G., 2011. Le statut social des enseignants français au prisme du renouvellement générationnel. Revue européenne des sciences sociales 49 (1), 157-178.

Freidson, E., 1984. La profession médicale. Payot, Paris [1970].

Freidson, E., 1986. Professional Powers: A Study of the Institutionalization of Formal Knowledge. University of Chicago Press, Chicago.

Gaïti, B., 1998. De Gaulle, prophète de la Cinquième République (1946-1962). Presses de Sciences Po, Paris.

Garcia, S., 2011. Mères sous influence. De la cause des femmes à la cause des enfants. La Découverte, Paris.

Garcia, S., Oller, A.C., 2015. Réapprendre à lire. De la querelle des méthodes à l'action pédagogique. Le Seuil, Paris.

Gossot, B., 1997. Les réseaux d'aide spécialisée aux élèves en difficulté : examen de quelques situations départementales. Rapport du Groupe de l'enseignement primaire, IGEN.

Guillarmé, J.-J., Luciani, D., 2011. La querelle des aides à l'école. Étude comparative et évaluation des résultats de « l'aide personnalisée à l'élève » et des « aides spécialisées » à l'enfant. Rapport de recherche, FNAREN.

Hughes, E.C., 1962. Good People and Dirty Works. Social Problems X (1), 3-11.

Hughes, E.C., 1996. Le regard sociologique. Essais choisis. Textes rassemblés et présentés par J.-M. Chapoulie. Éditions de l'EHESS, Paris.

Isambert-Jamati, V., 1985. Quelques rappels de l'émergence de l'échec scolaire comme « problème social » dans les milieux pédagogiques. In: Plaisance, E. (Ed.), L'échec scolaire. Nouveaux débats, nouvelles approches sociologiques. Éditions du CNRS, Paris, pp. 155-163.

Lascoumes, P., Le Gales, P., 2007. Sociologie de l'action publique. Armand Colin, Paris.

Le Bianic, T., 2013. Une profession balkanisée : les psychologues face à l'État en France (1945-1985). Politix 102, 175-207.

Mathieu-Fritz, A., Bercot, R., 2008. Le prestige des professions et ses failles. Hermann, Paris.

Matonti, F., Poupeau, F., 2004. Le capital militant. Essai de définition. Actes de la recherche en sciences sociales 155 , $5-11$.

Massardier, G., Verdier, É., 2005. L'évaluation pluraliste dans l'action publique. Espace temps 89/90, 79-86.

McAdam, D., 1988. Freedom Summer. Oxford University Press, New York.

Mingat, A., 1991. Les activités de rééducation GAPP à l'école primaire. Analyse du fonctionnement et évaluation des effets. Revue française de sociologie 32 (4), 515-549.

Millet, M., Thin, D., 2005. Ruptures scolaires. L'école à l'épreuve de la question sociale. Presses universitaires de France, Paris.

Morel, S., 2012. Les professeurs des écoles et la psychologie. Les usages sociaux d'une science appliquée. Sociétés contemporaines $85,133-160$.

Morel, S., 2014. La médicalisation de l'échec scolaire. La Dispute, Paris.

Offerlé, M., 1998. Sociologie des groupes d'intérêt. Montchrestien, Paris.

Pinell, P., Zafiropoulos, M., 1978. La médicalisation de l'échec scolaire. De la pédopsychiatrie à la psychanalyse infantile. Actes de la recherche en sciences sociales 24, 23-49.

Pinell, P., Zafiropoulos, M., 1983. Un siècle d'échecs scolaires. 1882-1982. Éditions sociales, Paris.

Sawicki, F., 2015. Esquisse d'une sociologie politique des enseignants français. Éducation et sociétés 36, 83-102. 
Schwartz, O., 1993. Postface. L'empirisme irréductible. In: Anderson, N. (Ed.), Le Hobo, sociologie du sans-abri. Nathan, Paris, pp. 265-305.

Simmel, G., 1992. Le conflit. Circé, Paris.

Van Zanten, A., 2001. L'école de la périphérie : scolarité et ségrégation en banlieue. Presses universitaires de France, Paris.

Verdier, É., 2012. Éducation. In: Bevort, A., Jobert, A., Lallement, M., Mias, A. (Eds.), Dictionnaire du travail. Presses universitaires de France, Paris, pp. 228-234. 